\title{
Advanced oxidation protein products induce intestine epithelial cell death through a redox-dependent, c-jun N-terminal kinase and poly (ADP-ribose) polymerase-1-mediated pathway
}

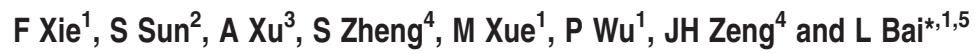

Advanced oxidation protein products (AOPPs), a novel protein marker of oxidative damage, have been confirmed to accumulate in patients with inflammatory bowel disease (IBD), as well as those with diabetes and chronic kidney disease. However, the role of AOPPs in the intestinal epithelium remains unclear. This study was designed to investigate whether AOPPs have an effect on intestinal epithelial cell (IEC) death and intestinal injury. Immortalized rat intestinal epithelial (IEC-6) cells and normal Sprague Dawley rats were treated with AOPP-albumin prepared by incubation of rat serum albumin (RSA) with hypochlorous acid. Epithelial cell death, nicotinamide adenine dinucleotide phosphate (NADPH) oxidase subunit activity, reactive oxygen species (ROS) generation, apoptosis-related protein expression, and c-jun N-terminal kinase (JNK) phosphorylation were detected both in vivo and in vitro. In addition, we measured AOPPs deposition and IEC death in 23 subjects with Crohn's disease (CD). Extracellular AOPP-RSA accumulation induced apoptosis in IEC-6 cultures. The triggering effect of AOPPs was mainly mediated by a redox-dependent pathway, including NADPH oxidase-derived ROS generation, JNK phosphorylation, and poly (ADP-ribose) polymerase-1 (PARP-1) activation. Chronic AOPP-RSA administration to normal rats resulted in AOPPs deposition in the villous epithelial cells and in inflammatory cells in the lamina propria. These changes were companied with IEC death, inflammatory cellular infiltration, and intestinal injury. Both cell death and intestinal injury were ameliorated by chronic treatment with apocynin. Furthermore, AOPPs deposition was also observed in IECs and inflammatory cells in the lamina propria of patients with CD. The high immunoreactive score of AOPPs showed increased apoptosis. Our results demonstrate that AOPPs trigger IEC death and intestinal tissue injury via a redox-mediated pathway. These data suggest that AOPPs may represent a novel pathogenic factor that contributes to IBD progression. Targeting AOPP-induced cellular mechanisms might emerge as a promising therapeutic option for patients with IBD.

Cell Death and Disease (2014) 5, e1006; doi:10.1038/cddis.2013.542; published online 16 January 2014

Subject Category: Experimental Medicine

The term inflammatory bowel disease (IBD) encompasses two major forms: ulcerative colitis and Crohn's disease (CD), both of which are characterized by chronic or recurrent relapsing gastrointestinal inflammation. ${ }^{1}$ Although a number of risk factors have been identified, IBD etiology and pathogenesis remain unclear. A peroxidation/antioxidation imbalance has been demonstrated in IBD development, 2,3 and this results in excessive reactive oxygen species (ROS) generation and oxidative stress. Such changes are able to induce the oxidative modification of proteins, thus causing structural and functional changes. ${ }^{4}$ The recently discovered advanced oxidation protein products (AOPPs) are dityrosinecontaining and cross-linking protein products formed during oxidative stress that are formed mainly by the reaction of plasma proteins with chlorinated compounds. ${ }^{5,6}$

Increased plasma AOPP formation has been reported in patients with chronic kidney disease, ${ }^{5}$ diabetes, ${ }^{7}$ and chronic hepatitis $C{ }^{8}$ As a novel protein marker of oxidant-mediated protein damage, AOPPs participate in these pathophysiologic conditions. They are capable of inducing vascular endothelial dysfunction via a receptor for advanced glycation endproducts (RAGE)-mediated signaling pathway. ${ }^{9}$ AOPPs have also been reported to induce overproduction of extracellular matrix and the fibrogenic factor transforming growth factor- $\beta 1$. Furthermore, Zhou et al. reported that AOPP accumulation promotes podocyte apoptosis and depletion through RAGE. ${ }^{10}$

\footnotetext{
${ }^{1}$ Guangdong Provincial Key Laboratory of Gastroenterology, Department of Gastroenterology, Nanfang Hospital, Southern Medical University, Guangzhou, China; ${ }^{2}$ Department of Hepatobiliary Surgery, Nanfang Hospital, Southern Medical University, Guangzhou, China; ${ }^{3}$ Huizhou Medical Institute, Huizhou, China; ${ }^{4}$ Department of Orthopedic and Spinal Surgery, Southern Medical University, Guangzhou, China and ${ }^{5}$ Department of Huiqiao Building, Southern Medical University, Guangzhou, China ${ }^{*}$ Corresponding author: L Bai, Department of Huiqiao Building, Nanfang Hospital, Southern Medical University, Guangzhou 510515, China. Tel: +86 20 61642251; Fax: +86 20 61642494; E-mail: bailan9@ 126.com

Keywords: AOPPs; intestine epithelial cell; death; redox; c-jun N-terminal kinase; PARP-1

Abbreviations: AIF, apoptosis-inducing factor; AOPPs, advanced oxidation protein products; CD, Crohn's disease; DPI, diphenylene iodinium; IBD, inflammatory bowel disease; IEC, intestinal epithelial cell; JNK, c-jun N-terminal kinase; PAR, polymers of ADP-ribose; PARP-1, poly(ADP-ribose) polymerase-1; PBS, phosphatebuffered saline; RAGE, receptor for advanced glycation end products; RSA, rat serum albumin; ROS, reactive oxygen species; TUNEL, terminal deoxynucleotidyl transferase dUTP nick-end labeling; UC, ulcerative colitis

Received 20.9.13; revised 04.12.13; accepted 05.12.13; Edited by A Stephanou
} 
Our recent study demonstrated that AOPPs inhibit the proliferation and differentiation of rat osteoblast-like cells via ROS generation and nuclear factor- $\kappa$ B signaling. ${ }^{11}$

Intestinal epithelial cells (IECs) are organized as a single cell layer that forms a contiguous lining and functional barrier that maintains gut structural integrity to separate the bowel wall from microbes and toxins. ${ }^{12,13}$ IEC proliferation and death must be tightly regulated to maintain the structural integrity of the intestinal mucosal epithelium, and changing this balance can have pathological consequences. There is a growing body of literature showing that excessive cell death is associated with chronic inflammation, as seen in patients with IBD, and this could contribute to IBD pathophysiology. ${ }^{14,15}$ Two major cell death pathways, the caspase-3 pathway and the recently identified caspase-independent pathway mediated by the activation of poly (ADP-ribose) polymerase-1 (PARP-1), lead to apoptotic cell death following ischemia, inflammatory injury, and ROS-induced injury. ${ }^{15,16}$ Although previous studies have revealed that oxidative stress results in plasma accumulation of AOPPs in IBD, ${ }^{17,18}$ the effects of AOPPs on IECs remain unclear. It is unknown whether AOPPs affect IEC proliferation and death or intestinal tissue injury. Moreover, there is no information regarding the possible deposition of AOPPs in the intestinal tissue of patients with IBD. In the present study, we determined the effects of AOPPs on IEC death both in vitro and in vivo and investigated the cellular pathway underlying the pro-apoptotic effect of AOPPs.

\section{Results}

Increased extracellular AOPPs triggered IEC apoptosis in vitro. To determine whether AOPPs accumulation induces IEC apoptosis, we subjected conditionally immortalized IEC- 6 cultures to increasing concentrations of AOPP-rat serum albumin (RSA) for $48 \mathrm{~h}$ or $200 \mu \mathrm{g} / \mathrm{ml}$ of AOPP-RSA for increasing times. Healthy IEC- 6 cultures contained intact nuclei, but AOPP-RSA-treated cells exhibited nuclear condensation followed by fragmentation (Figure 1a). Quantitative fluorescence-activated cell sorting (FACS) analysis of fluorescein isothiocyanate (FITC)-annexinV/propidium iodide $(\mathrm{PI})$ staining showed that AOPP-RSA caused IEC-6 apoptosis in a concentration- and timedependent manner compared with cells cultured in control medium and treated with unmodified RSA (Figures $1 b-d$ ).

\footnotetext{
AOPP-triggered apoptosis was mediated by NADPH oxidase-dependent ROS production. Previous studies demonstrated that intracellular ROS mediate AOPP-induced podocyte and mesangial cell apoptosis. ${ }^{10}$ Therefore, we examined intracellular ROS levels in AOPP-treated IEC-6 cultures; dichlorofluorescein (DCF) fluorescence in the FITC/FL-1 channel was used to assess ROS generation. As shown in Figure 2a, incubation of IEC-6 cultures with AOPP-RSA induced time- and dose-dependent increases in ROS production. To evaluate whether nicotinamide adenine dinucleotide phosphate (NADPH) oxidases were responsible for intracellular ROS generation, the experiment was repeated with the NADPH oxidase inhibitors diphenylene iodinium (DPI) and apocynin. AOPP-induced ROS generation was
}

significantly decreased in IEC-6 cultures that were pretreated with superoxide dismutase (SOD), DPI, or apocynin separately (Figure $2 \mathrm{~b}$ ). We also evaluated NADPH oxidase activity in IEC-6 cultures stimulated with AOPP-RSA. As shown in Figure 2, treatment with AOPPs led to membrane translocation (Figure 2c) and phosphorylation of $\mathrm{p} 47^{\text {phox }}$ (Figure 2d), as well as increased expression levels of NADPH oxidase key components $\mathrm{p} 22^{\text {phox }}, \mathrm{p} 47^{\text {phox }}$, and gp91 ${ }^{\text {phox }}$ (Figure 2e). These results suggested that AOPPtriggered ROS production was dependent on cellular NADPH oxidase activation in IEC-6 cultures.

Next, we sought to elucidate the role of ROS and NADPH oxidase in AOPP-induced apoptosis. In IEC-6 cultures treated with $200 \mu \mathrm{g} / \mathrm{ml}$ AOPPs in the presence of the general ROS scavenger SOD, AOPP-triggered apoptosis was largely abolished (Figure 2f). Similarly, inhibition of NADPH oxidase with apocynin and DPI significantly reduced IEC-6 apoptosis induced by AOPPs (Figure 2f). Taken together, these findings imply that AOPPs are sufficient to induce IEC-6 apoptosis by increasing ROS synthesis, which is mediated through cellular NADPH oxidase activation.

AOPP-triggered apoptosis was associated with JNK activation. Intracellular mitogen-associated protein kinases (MAPKs), including extracellular-signal regulating kinase 1/2 (ERK1/2 or p44/42 MAPK), c-jun N-terminal kinase (JNK), and p38 MAPK, have been shown to regulate cell growth, death, and cellular responses to stress. ${ }^{19}$ To determine whether the MAPK pathway is involved in AOPP-RSAtriggered cell death, we examined MAPK activity in IEC-6 cultures treated with AOPPs. As illustrated in Figure 3a, JNK phosphorylation was markedly increased from 30 to $120 \mathrm{~min}$ after AOPPs treatment. However, AOPPs had no significant effect on phospho-p38 or phospho-ERK1/2 MAPK levels (data not shown).

AOPPs-activated PARP-1 via the NADPH oxidase-ROSJNK pathway. It is reported that the caspase-3 and caspase-independent (mediated by PARP-1 activation) pathways can both lead to cell death after inflammatory injury ${ }^{14,20}$ or ROS-induced injury. ${ }^{16}$ The former is the classic pathway marked by degradation of procaspase-3 into cleaved caspase-3. The latter is characterized by the formation of polymers of ADP-ribose (PAR), decreased NAD + levels, cytosolic apoptosis-inducing factor (AIF) nuclear translocation, nuclear condensation, and cell death. ${ }^{16}$ To confirm which was involved in AOPP-induced death, we examined the activities of both pathways in IEC- 6 cultures incubated with AOPP-RSA. We verified that AOPPs stimulated robust PARP-1 activation in IEC-6 cultures from $1 \mathrm{~h}$, which was accompanied by PAR formation (Figure $3 b$ ) and $\mathrm{NAD}^{+}$ decrease (Figure 3c) and was followed by AIF nuclear translocation from $6 \mathrm{~h}$ on (Figure 4). Interestingly, decreased procaspase-3 protein and increased cleaved caspase-3 could be detected after AOPPs treatment (Figure 3b).

To further evaluate the role of JNK-MAPK in cell apoptosis, IEC-6 cultures were incubated with a JNK inhibitor (SP600125) before AOPP-RSA treatment. The results suggested that activation of the proapoptotic JNK-MAPK pathway has a critical role in AOPP-induced IEC-6 apoptosis. 


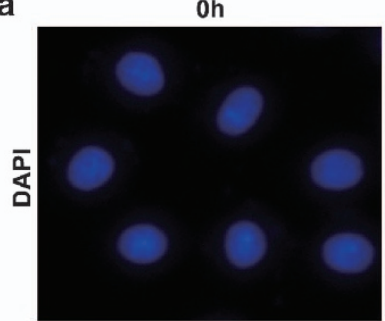

b

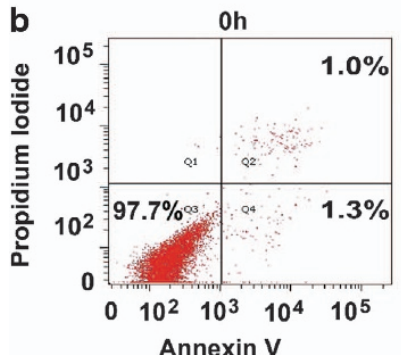

Annexin V
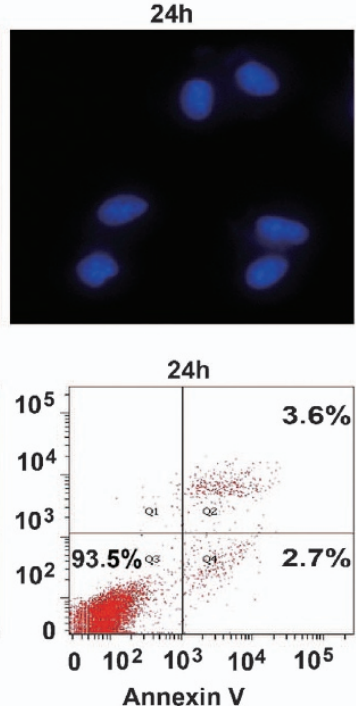

$48 \mathrm{~h}$
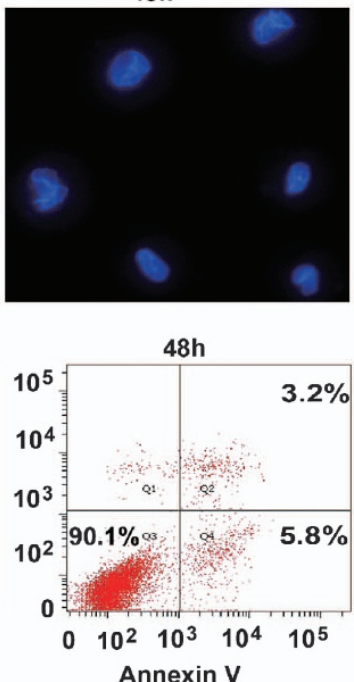

$72 \mathrm{~h}$
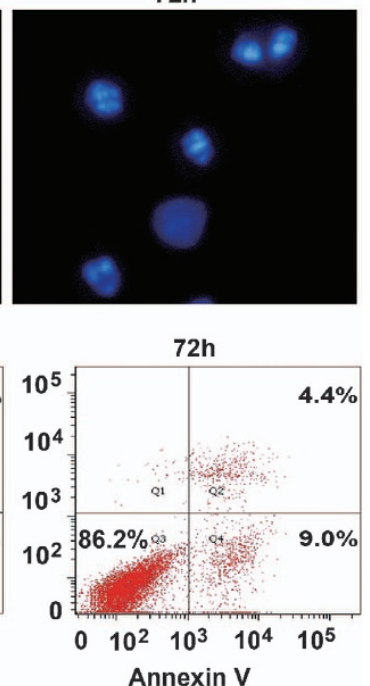

C
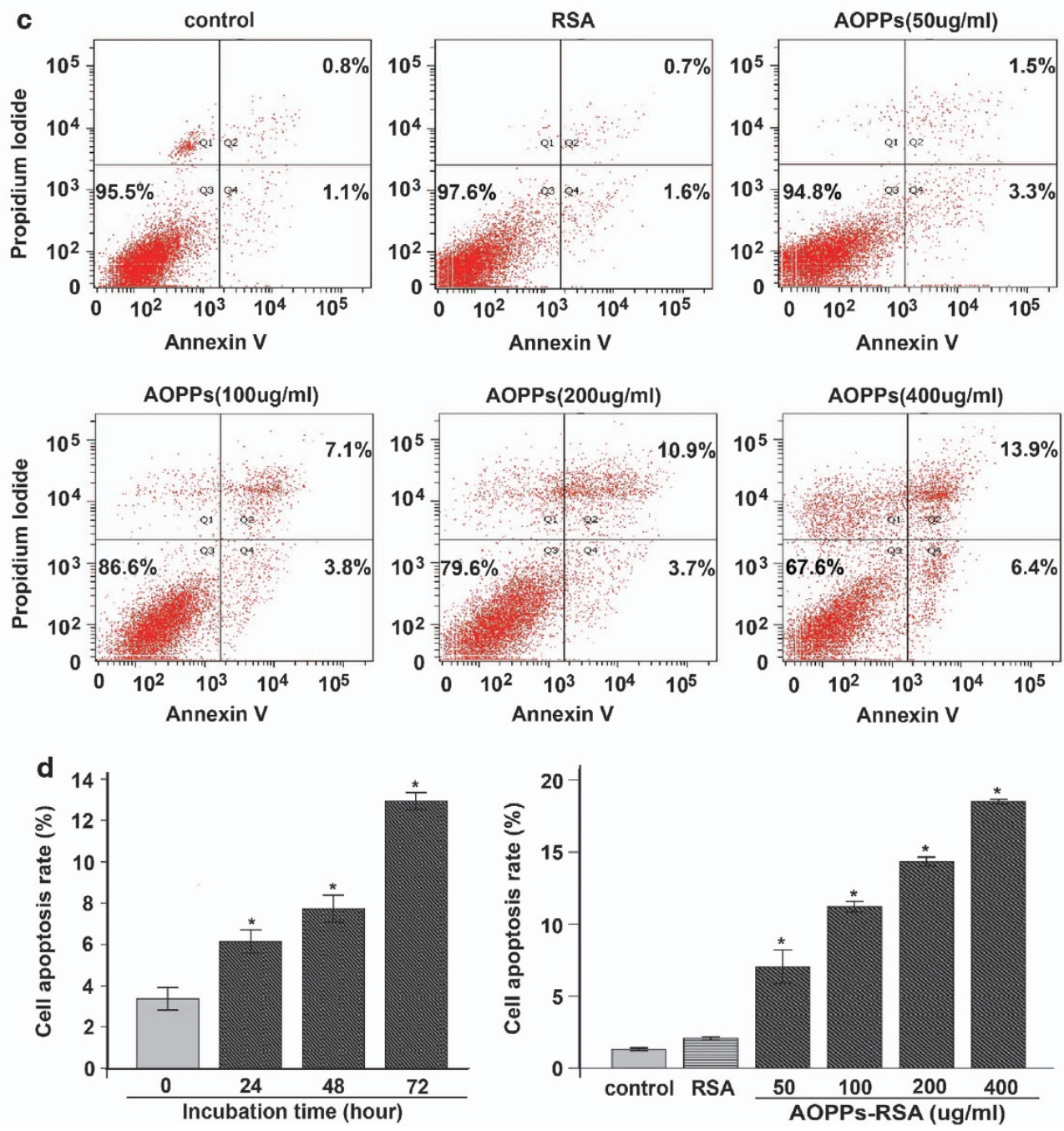

Figure 1 AOPPs challenge induced IEC apoptosis in a concentration- and time-dependent manner. (a) Apoptotic morphology of IEC-6 cells nuclei. The nuclear condensation/fragmentation observed with DAPI staining after AOPP-RSA treatment. (b-c) Representative dot blots of FITC-annexin-V versus PI. IEC-6 cells were incubated with $200 \mu \mathrm{g} / \mathrm{ml}$ AOPP-RSA for the indicated time period, or the indicated concentrations of AOPP-RSA or native RSA for $24 \mathrm{~h}$. Apoptosis was quantified by measuring combined early and late apoptotic cells using flow cytometry and was found to increase in a time- and dose-dependent manner. ${ }^{*} P<0.01$ versus control. (d) Histogram of total FITC-annexin-V fluorescence (inset). Data are presented as mean \pm S.D. from experiments performed in triplicate. ${ }^{*} P<0.05$ versus control 

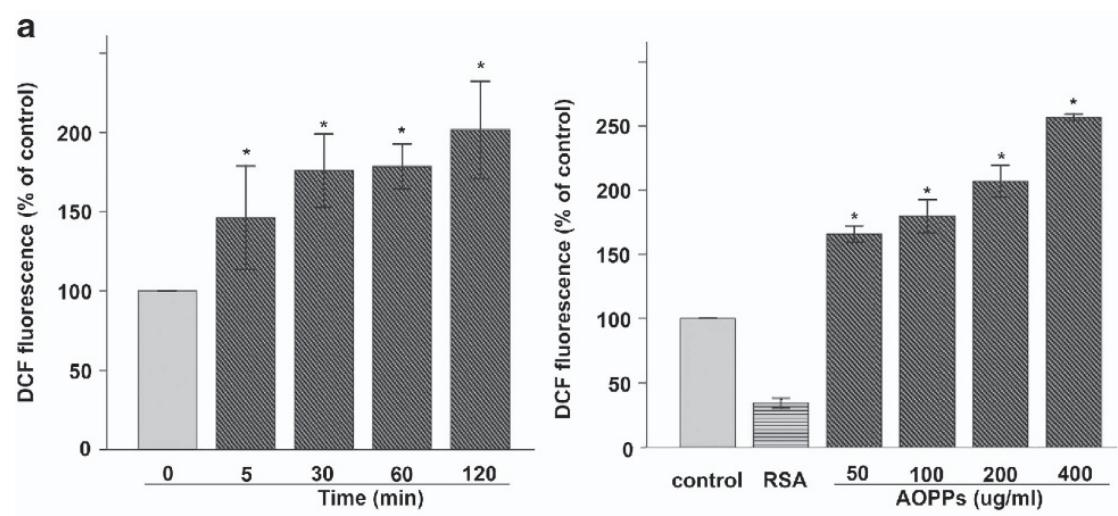

b
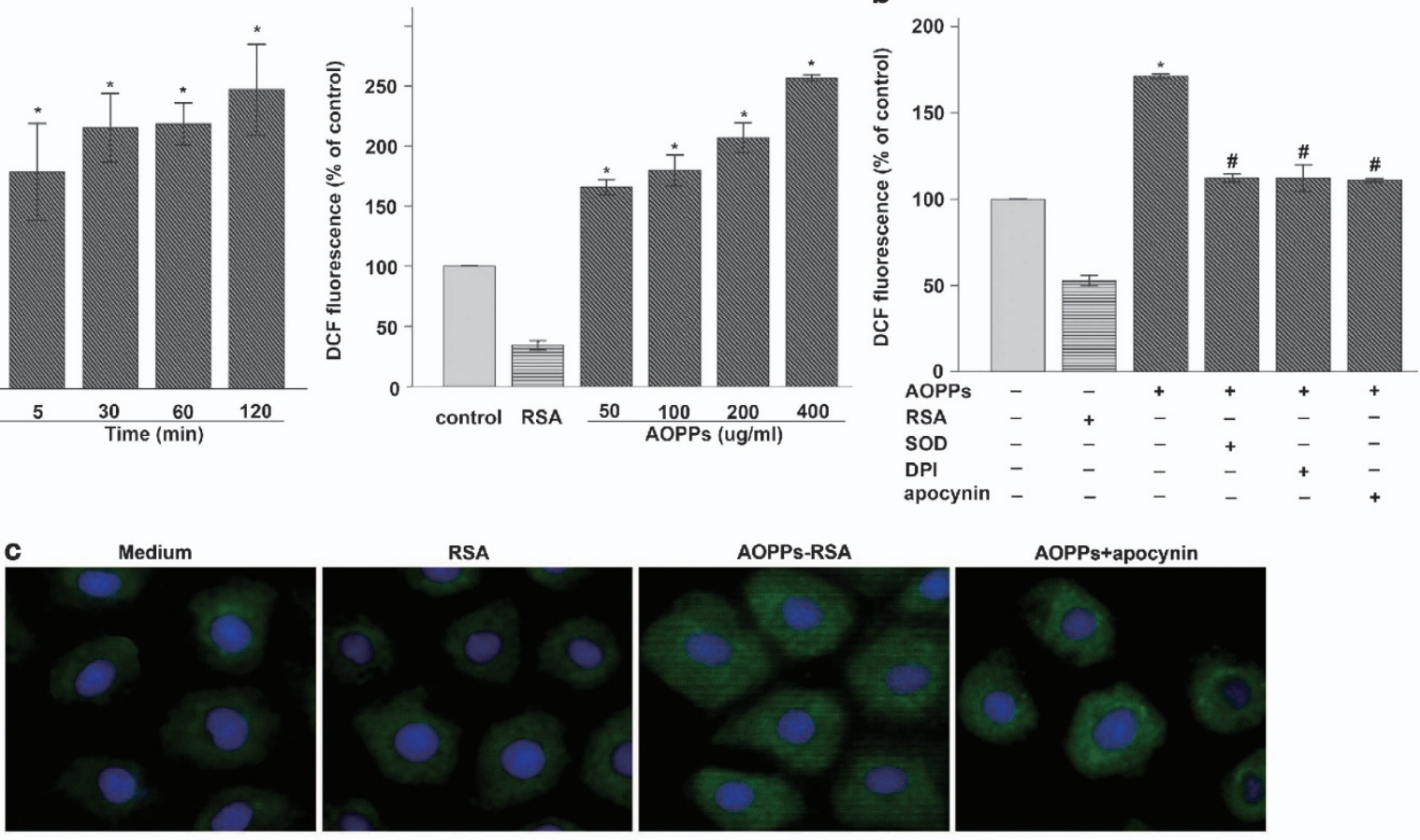

d

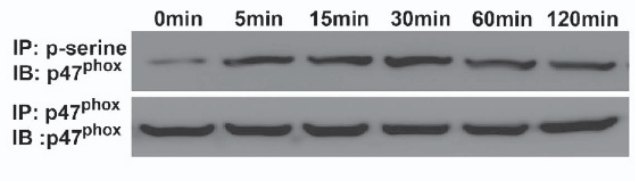

e
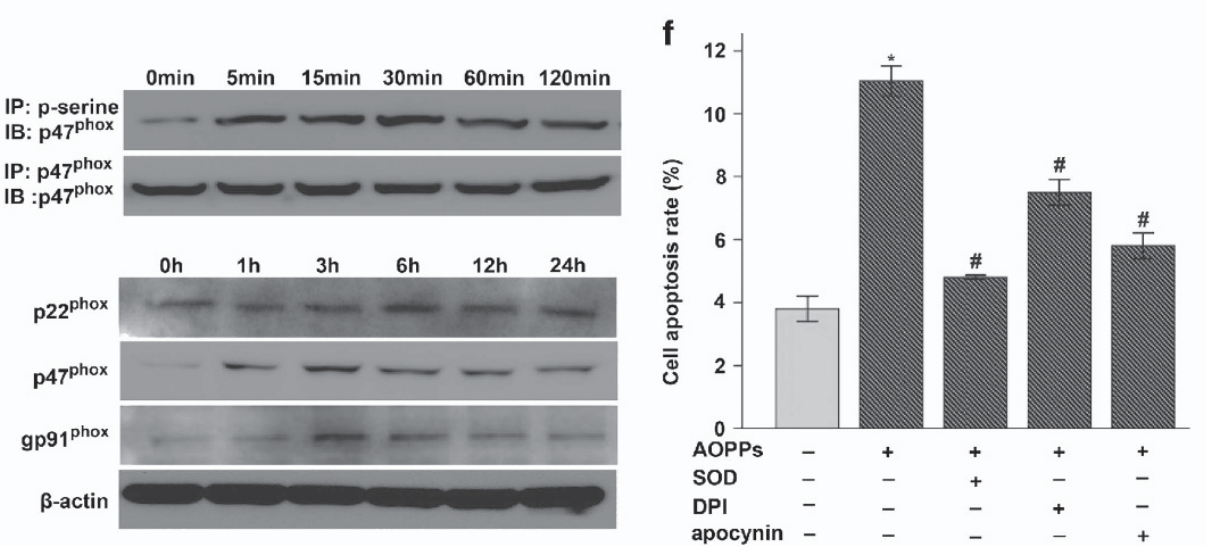

Figure 2 AOPPs triggered intracellular NADPH oxidase-derived ROS production in IEC-6 cells. (a) IEC-6 cells were incubated with control medium, RSA, or AOPPs prior to a 30-min DCFH-DA treatment. ROS production was determined by flow cytometry quantification of DCF fluorescence. Data are presented as mean \pm S.D. from experiments performed in triplicate. ${ }^{*} P<0.05$ versus control. (b) IEC- 6 cells were incubated with AOPPs in the presence or absence of SOD, DPI, or apocynin for the indicated times, and AOPP-triggered ROS generation was significantly decreased by pretreatment with NADPH oxidase inhibitors, as well as SOD. (c) Representative images of AOPP-induced membrane translocation of $p 47^{\text {phox }}$. Magnification is $\times 400$. (d) Co-immunoprecipitation showed $p 47^{\text {phox }}$ phosphorylation. (e) AOPP-induced activation of NADPH oxidase in IEC-6 cells. IEC-6 cells were incubated with AOPPs for 0-24h, and protein expression levels of NADPH oxidase subunits, including p47 ${ }^{\text {phox }}$, p22 $^{\text {phox }}$, and gp91 ${ }^{\text {phox }}$, were determined by western blotting. (f) IEC-6 cells were pretreated with a ROS scavenger (SOD) and NADPH oxidase inhibitors (DPI and apocynin), The cells were then treated with $200 \mu \mathrm{g} / \mathrm{ml}$ AOPP-RSA for $24 \mathrm{~h}$. Apoptosis was quantified by flow cytometry. Data are presented as the mean \pm S.D. of three experiments. ${ }^{*} P<0.05$ versus control. ${ }^{\#} P<0.05$ versus AOPPs

To further determine the roles of JNK, PARP-1, and caspase-3 in AOPP-induced apoptosis, IEC-6 cultures were incubated with a JNK inhibitor (SP600125), the PARP-1 inhibitor 3,4-dihydro-5-[4-(1-piperidinyl)butoxy]-1 $(2 \mathrm{H})$-isoquinolin-one (DPQ), or the broad-spectrum caspase inhibitor Z-VAD.fmk before AOPP-RSA stimulation. SP600125 almost completely abolished the AOPP-induced increase in cell apoptosis. DPQ significantly decreased AOPP-triggered cell apoptosis. However, caspase inhibitor treatment failed to statistically decrease AOPP-induced toxicity (Figure 3d). These data indicate that AOPP-induced cell death is dependent on activation of the proapoptotic JNK-MAPK and PARP-1 pathway, not caspase-3 signaling.

We also pre-treated IEC-6 cultures with DPI, apocynin SOD, or SP600125 before AOPP-RSA incubation. We found that PARP-1 activation was significantly suppressed by SOD, DPI, apocynin, and especially by SP600125. Over time, these suppressive effects became more obvious (Figure 3e). Therefore, we concluded that AOPPs activate PARP-1 via an NADPH-dependent ROS-JNK pathway. 

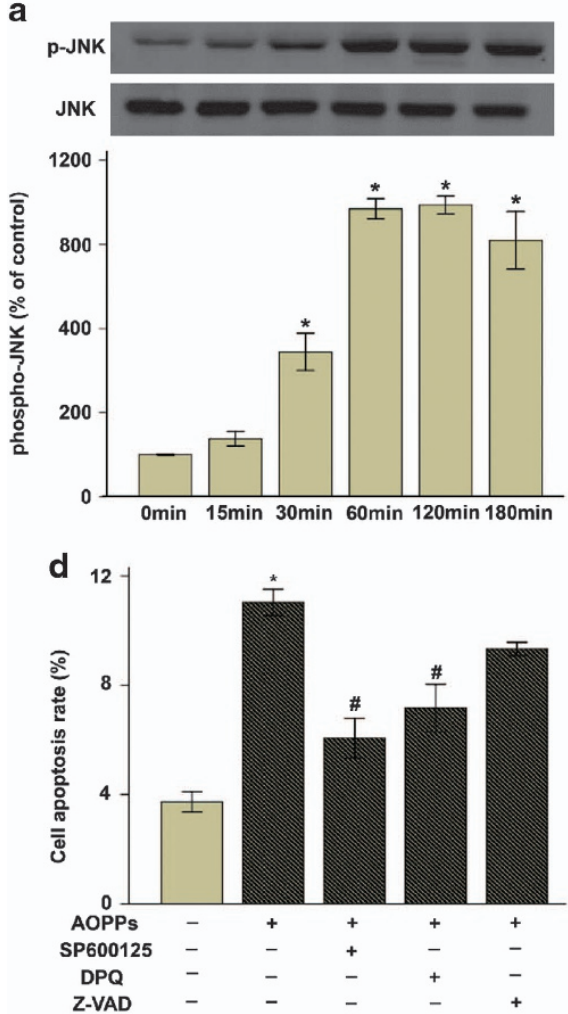
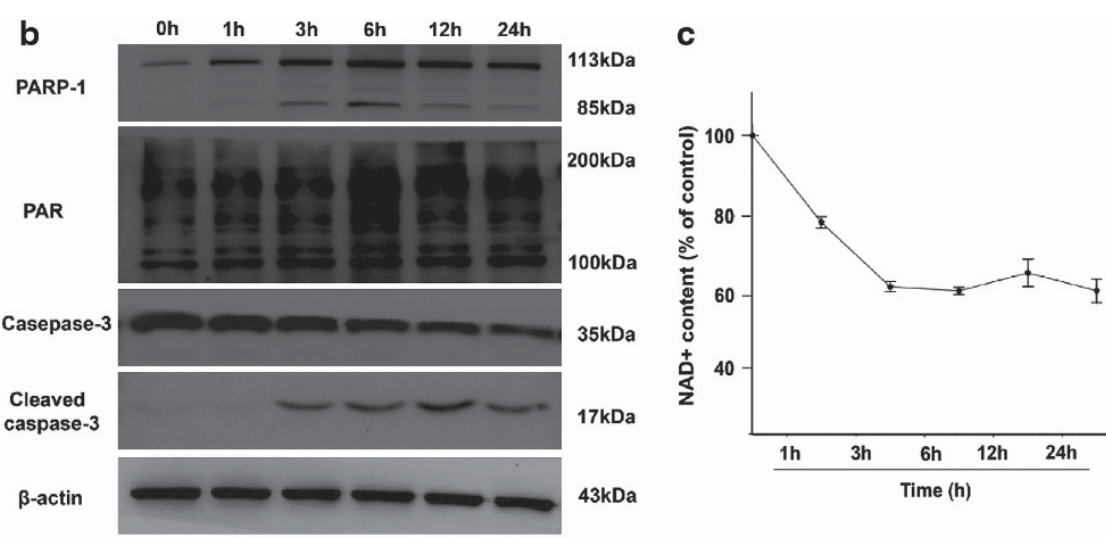

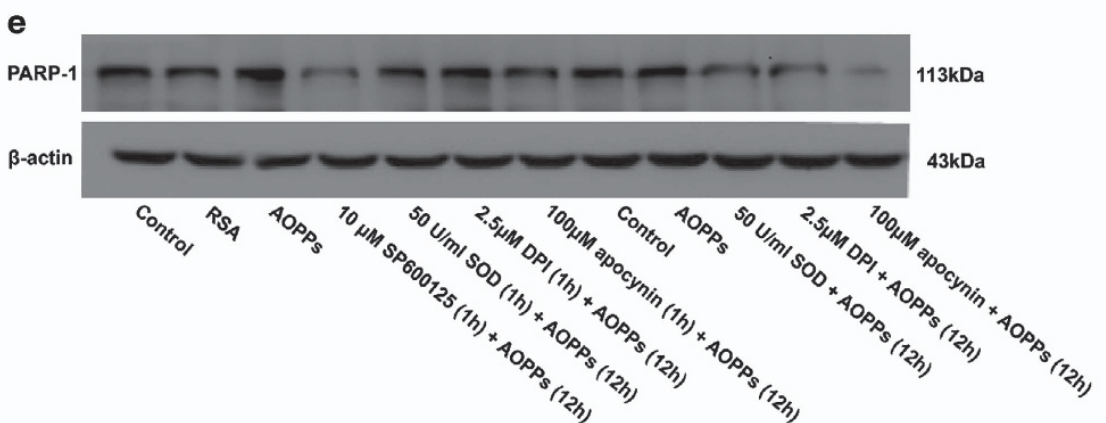

Figure 3 Cellular events after AOPPs treatment. (a) p-JNK activation in AOPP-treated IEC-6 cells. (b) AOPP challenge induced PARP-1 activation and PAR formation in parallel with a reduction of nicotinamide adenine dinucleotide $(N A D+)$ as shown in Figure $3 \mathrm{c}$. Caspase-3 was activated from $3 \mathrm{~h}$ post-AOPP treatment, at the same time PARP-1 cleavage was observed. (c) Time-course analysis of cellular NAD + depletion in IEC- 6 cells after AOPP treatment. NAD + level reduced to $80 \%$ of control within $1 \mathrm{~h}$, and was maintained at $67 \%$ after $3 \mathrm{~h}(P<0.001)$. (d) IEC-6 cells were pretreated with a JNK inhibitor (SP600125), a PARP inhibitor (DPQ), or a caspase-3 inhibitor before AOPP-RSA incubation. SP600125 and DPQ significantly decreased AOPP-induced cell apoptosis, but Z-VAD failed. (e) AOPP-induced PARP-1 activation was inhibited by pre-incubation of SP600125, SOD, DPI, and apocynin. After $1 \mathrm{~h}$ pretreatment with SP600125, SOD, DPI, or apocynin, the cells were removed from or continuously exposed to these inhibitors, then the cells were treated with AOPPs for $12 \mathrm{~h}$. ${ }^{*} P<0.05$ versus control. ${ }^{\#} P<0.05$ versus AOPPs

IEC death was aggravated in AOPP-treated rats and relieved by apocynin. In an attempt to examine if the effects of AOPPs on cell death observed in vitro might also occur in vivo, normal male Sprague Dawley rats were randomly assigned into four groups and received intraperitoneal injections of normal saline, RSA, AOPP-RSA, or AOPP-RSA every other day with or without intragastric administration of apocynin for 12 weeks. We found that plasma AOPPs levels increased $\sim 0.5$-fold in AOPP-RSAtreated rats in comparison with control rats, which is equivalent to the level detected in patients with active CD (Table 1).

Terminal deoxynucleotidyl transferase dUTP nick-end labeling (TUNEL) staining revealed that IEC death was significantly aggravated in AOPP-treated rats when compared with that in control (vehicle- or RSA-treated rats) (Figure 5). Inhibition of NADPH oxidase by apocynin significantly ameliorated AOPP-induced cell death (Figure 5).

In vivo AOPP-triggered cell death was mediated by the NADPH oxidase-JNK-PARP-1 pathway. Immunohistochemical staining of intestine showed significant upregulations of $\mathrm{p} 47^{\text {phox }}$, gp9 $91^{\text {phox }}$, and $\mathrm{p} 22^{\text {phox }}$ in AOPPs-challenged rats compared with controls (Figure 6a). Western blotting confirmed increases in $\mathrm{p} 47^{\text {phox }}, \mathrm{gp} 91^{\text {phox }}$, and $\mathrm{p} 22^{\text {phox }}$ expression levels (Figure 6b). We also performed immunohistochemistry to demonstrate increased JNK phosphorylation and PARP-1 expression in AOPP-challenged rats. PAR generation and AIF translocation were also detected after AOPPs treatment (Figure 7). Moreover, IECs were positive for TUNEL but negative for caspase-3 (data not shown). These data provide further evidence that AOPP-triggered cell death in vivo is mediated by activation of the NADPH oxidase-JNK-PARP-1-PAR pathway rather than by caspase3 signaling. Treatment with apocynin significantly decreased AOPP-induced activation of the NADPH oxidase-JNKPARP-1-PAR pathway (Figures 6 and 7).

Chronic AOPPs administration promoted inflammation and injury in rat intestinal mucosa. Histological examination of the small intestine revealed that AOPPs were predominantly deposited in the crypts and lymphocytes of the lamina propria and villous epithelial cells (Figure 6). Systematic histological assessment of the intestinal tracts revealed significant inflammatory changes; these alterations were primarily localized to the terminal ileum and barely 
a
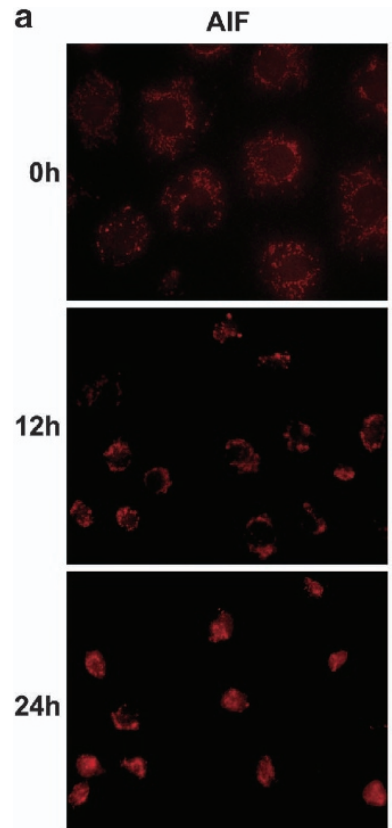
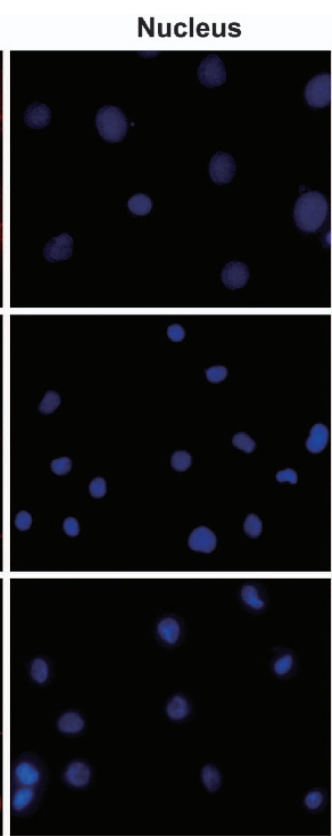
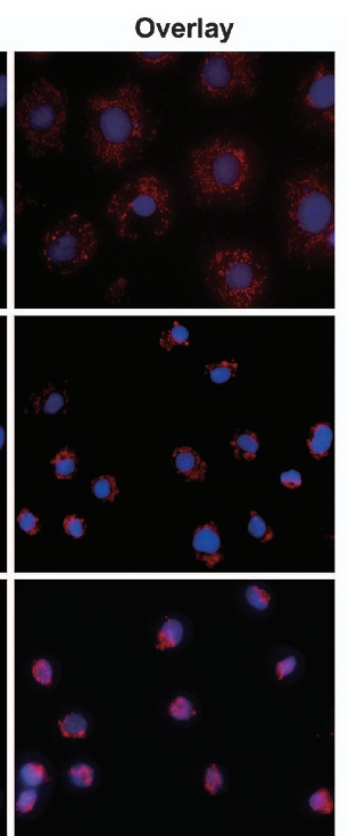

b

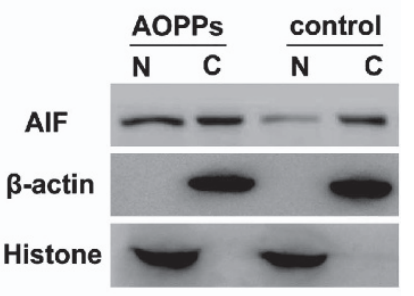

Figure 4 AIF translocation in AOPP-treated IEC-6 cells. (a) IEC-6 cells were incubated with an anti-AIF antibody after AOPP-RSA treatment for the indicated time, incubated with a rhodamine-conjugated secondary antibody, and counterstained with DAPI. AIF nuclear translocation is demonstrated by the overlap of AIF and nuclear staining. (b) Analysis of AIF translocation using nuclear/cytosolic fractionation immunoblotting. IEC-6 cells treated with AOPPs for $12 \mathrm{~h}$ were subjected to subcellular fractionation, and immunoblotting was performed with nuclear and cytosolic fractions. Histone and $\beta$-actin were used as nuclear and cytosolic marker proteins, respectively

Table 1 Body weight, plasma AOPPs, and histologic findings in rats

\begin{tabular}{lccc}
\hline Week 12 $(\boldsymbol{n}=\mathbf{6})$ & Body weight $(\mathbf{g})$ & Plasma AOPPs $(\boldsymbol{\mu M})$ & Inflammatory infiltrate $(\boldsymbol{n})$ \\
\hline Control & $335.22 \pm 35.22$ & $116.12 \pm 4.40$ & 0 \\
RSA & $328.83 \pm 28.83$ & $117.40 \pm 10.95$ & 0 \\
AOPPs & $318.36 \pm 18.36$ & $165.61 \pm 8.71^{*}$ & 0 \\
AOPPS + apocynin & $328.37 \pm 28.37$ & $142.91 \pm 14.02^{*} \#$ & 4 \\
\end{tabular}

Abbreviations: AOPPs, advanced oxidative protein products; RSA, rat serum albumin

${ }^{\star} P<0.05$ versus vehicle. ${ }^{\#} P<0.05$ versus AOPPs

Data are expressed as mean \pm S.D., $n=6$

infiltrated the colon tissue. The lesions consisted of shortened intestinal villous; lamina propria and submucosal infiltration of lymphocytes, plasmacytes, and scattered neutrophils; lymphoid follicle hyperplasia; epithelial necrosis and exfoliation; and erosion of the intestinal mucosal layer (Figures $8 \mathrm{c}-\mathrm{f}$ ). Apocynin treatment attenuated the degree of tissue injury (Figure 8h). In addition, periodic acid Schiff (PAS) staining showed that chronic AOPPs administration significantly decreased the number of goblet cells compared to control (Figures $8 \mathrm{i}-\mathrm{j}$ ). We also found that goblet cell numbers were reduced in both the crypts and villi, especially the latter (Figures $8 \mathrm{i}-\mathrm{j}$ ).

Intestinal mucosa AOPPs deposition was associated with cell death in CD patients. A previous study demonstrated that plasma AOPP concentrations were elevated in patients with IBD, particularly in those with active CD. ${ }^{17}$ To further evaluate the effects of AOPPs on IECs in patients with $C D$, we examined AOPP expression and cell death by immunohistochemistry and TUNEL staining, respectively, in sequential sections of intestinectomy specimens from 23 patients. The normal intestinal tissues adjacent to the diseased regions were used as normal control samples. As shown in Figure 9a, AOPPs were predominantly deposited in IECs and inflammatory cells in the lamina propria of intestinectomy specimens, whereas AOPPs staining was negative in normal intestinal tissue (Figure 9a). Likewise, TUNEL-positive cells were detected in the diseased region but rarely in the adjacent normal region (Figure 9b). In addition, the high immunoreactive score of AOPPs indicated increased cell death (Figure 9c), suggesting that AOPPs accumulation is associated with cell death in patients with CD.

\section{Discussion}

The formation and accumulation of plasma AOPPs are well demonstrated in diverse diseases. ${ }^{9,11,21}$ Increasing evidence suggests that AOPPs are pathogenic mediators participating in these disorders, which highlights the urgent need to understand their effects on cells, tissues, and organs under physiological and pathological conditions. In bowel diseases, 

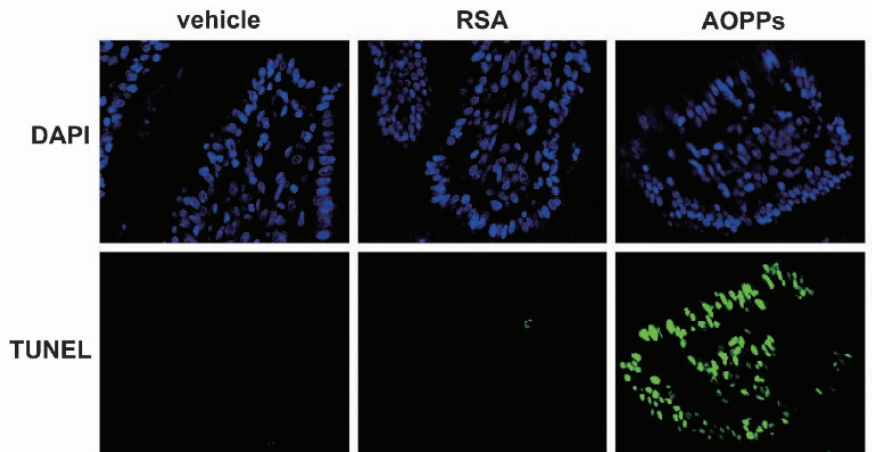

AOPPs+apocynin
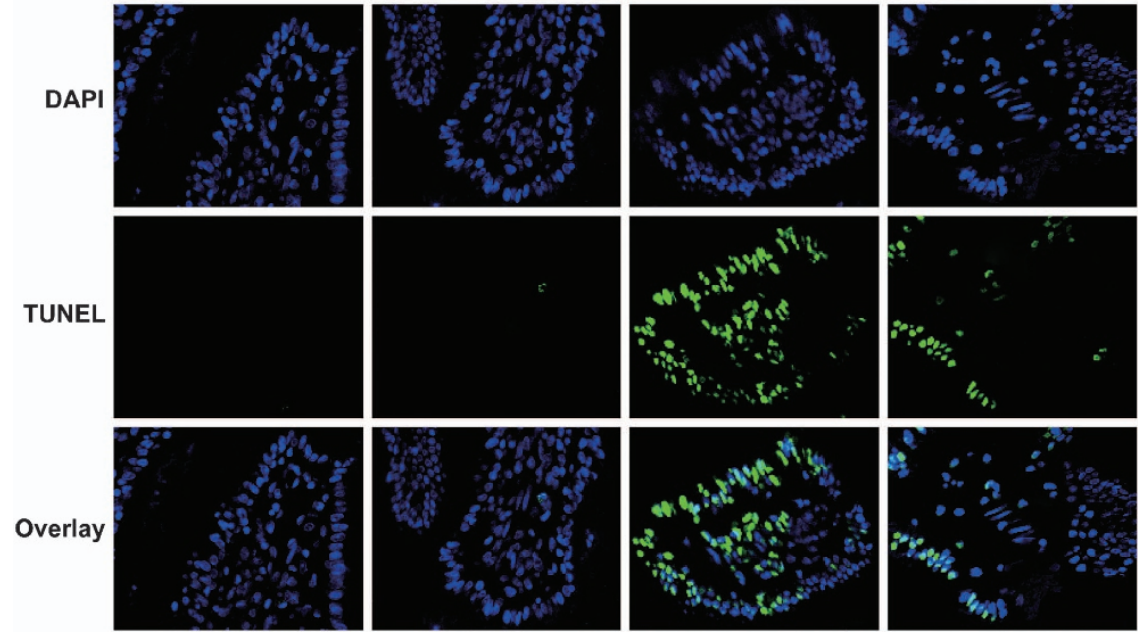

Figure 5 TUNEL staining. Representative photographs showing TUNEL immunofluorescence in rat small intestinal epithelium with or without AOPP-RSA treatment

a
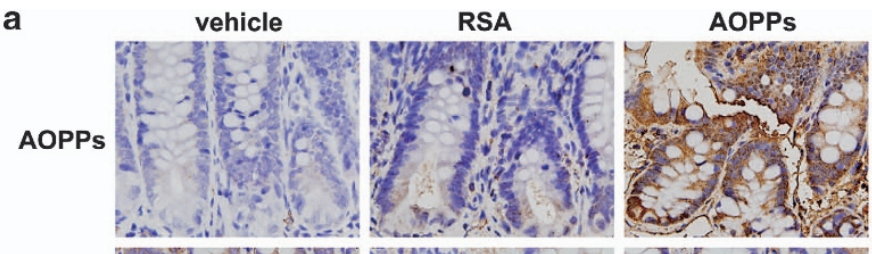

AOPPstapocynin
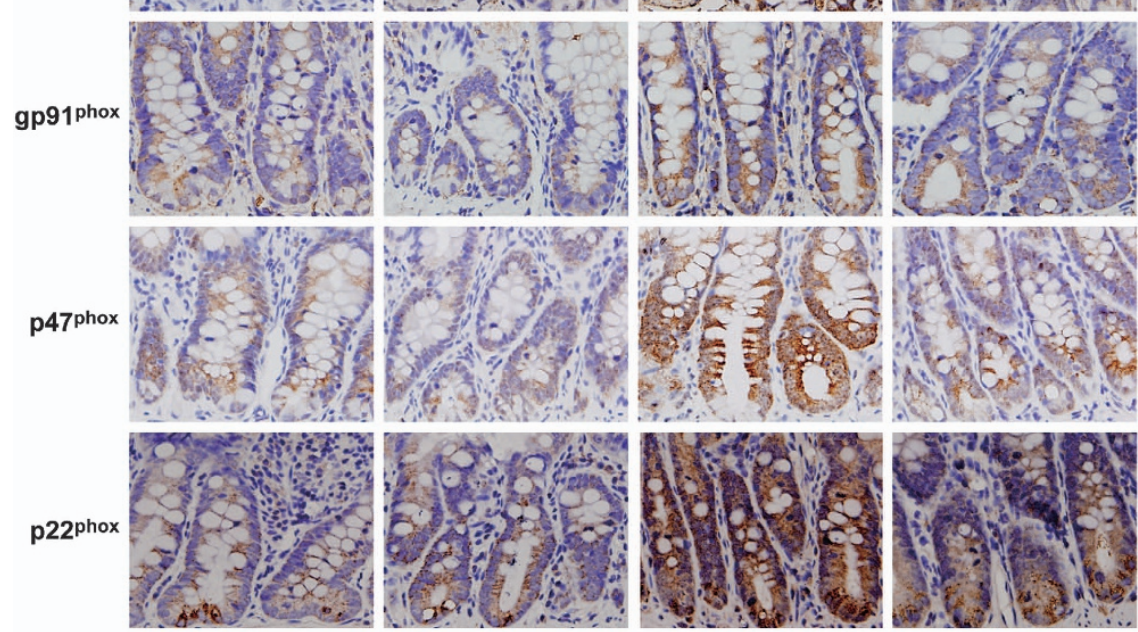

b
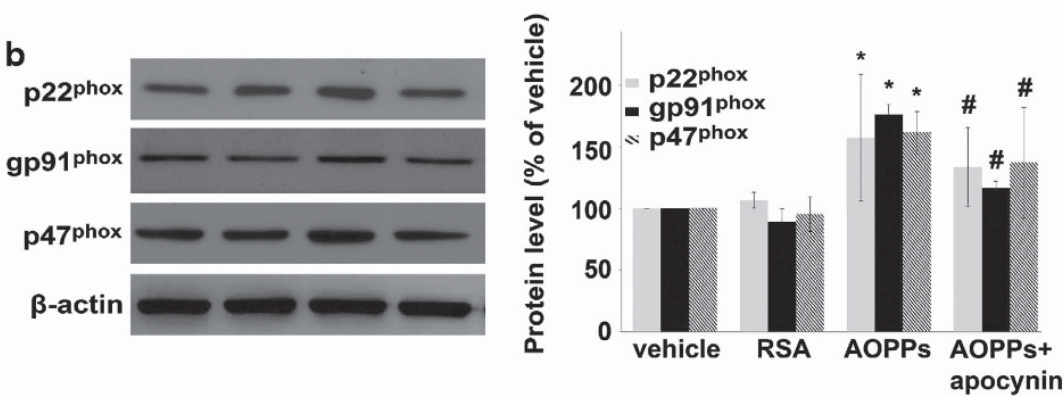

Figure 6 NADPH oxidase activation in AOPP-challenged rats. (a) Immunohistochemical staining of AOPPs, p22 $2^{\text {phox }}$, p4 $7^{\text {phox }}$, and gp9 ${ }^{\text {phox }}$ in AOPP-challenged rats and controls. (b) Expression levels of p22 ${ }^{\text {phox }}$, p47 ${ }^{\text {phox }}$, and gp91 ${ }^{\text {phox }}$ in intestinal mucosa were detected by western blotting. ${ }^{*} P<0.05$ versus vehicle. ${ }^{\#} P<0.05$ versus AOPPs

IEC death is a hallmark of intestinal chronic inflammation and has been proposed as a potential pathogenic mechanism driving IBD in humans. ${ }^{15}$ However, the regulation of IEC death remains poorly understood. In the present study, we present in vitro and in vivo lines of evidence that AOPPs induced IEC death via a redox-dependent, JNK- and PARP-1-mediated 


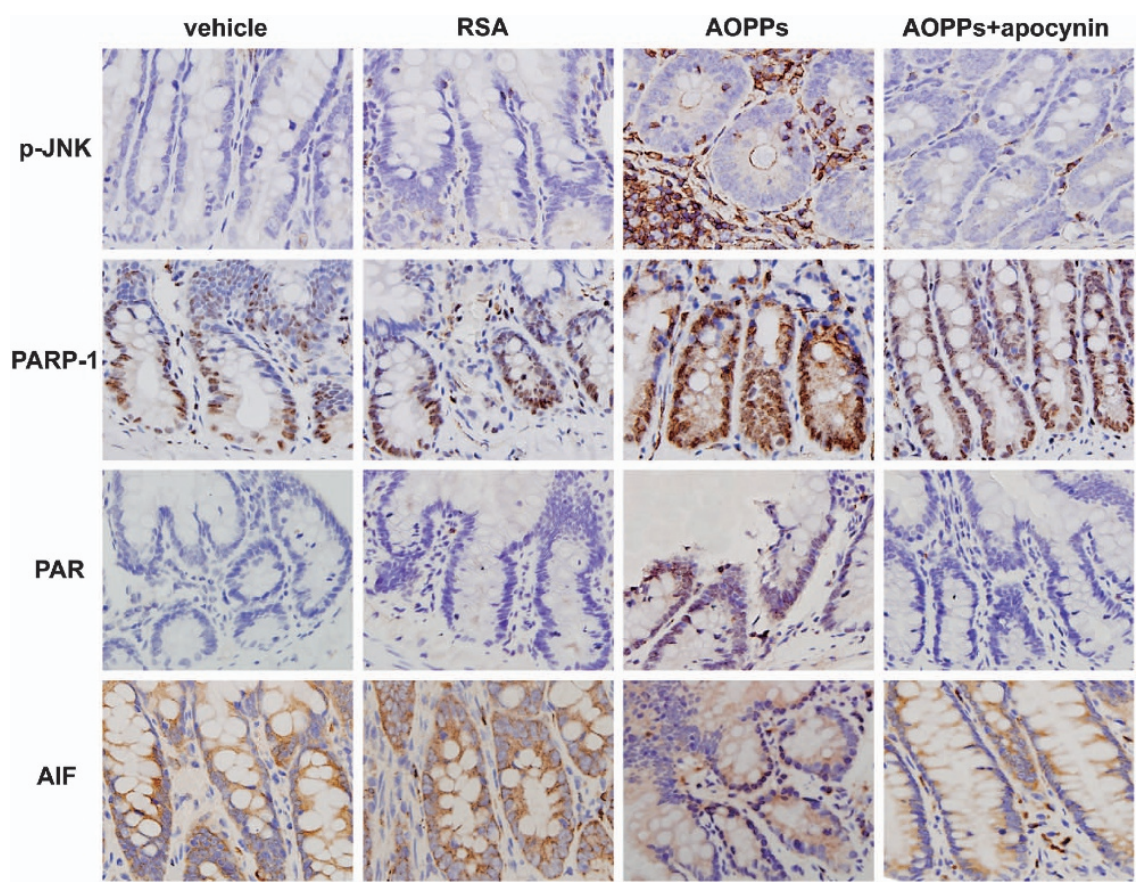

Figure 7 Immunohistochemical detection of p-JNK, PARP-1, PAR, and AIF in rat intestinal mucosa. AOPPs treatment increased JNK phosphorylation and PARP-1 expression. PAR generation and AIF translocation were detected in AOPP-challenged rats and was ameliorated by apocynin treatment

pathway. Chronic AOPP-RSA administration to normal rats led to AOPPs deposition in IECs, intestinal epithelial inflammation, and tissue injury. Although the AOPPs used in our study were prepared in vitro by incubating albumin with hypochlorous acid (HOCL), previous studies have demonstrated that the biological effects of AOPPs prepared by this method are similar with those extracted from patients. ${ }^{10}$ In addition, we found enhanced deposition of AOPPs in diseased regions, and their levels were associated with cell death in patients with CD. To the best of our knowledge, these lines of evidence are the first to verify AOPPs accumulation as a novel mechanism for IEC death and to demonstrate the pathogenic effect of AOPPs on intestinal epithelium. Collectively, they suggest that AOPPs might be involved in IBD progression by inducing IEC death and tissue injury.

Reports on the underlying mechanisms of AOPP-induced cell death are rare. Previous studies have described the involvement of NADPH oxidase-dependent ROS in AOPPinduced podocyte apoptosis. ${ }^{21}$ Therefore, to confirm that this mechanism was involved in IEC death, we assessed NADPH oxidase activity and ROS generation in immortalized IEC-6 cultures. The in vitro results confirmed that intestinal NADPH oxidases contribute to ROS production after AOPPs administration. Similar results were also observed in the AOPPtreated animal model. Interestingly, ROS production was greatly reduced after RSA treatment with respect to controls, suggesting that unmodified RSA may decrease ROS levels. MAPK signaling has been identified as a significant ROSsensitive signal transduction pathway associated with IEC proliferation and apoptosis. ${ }^{22}$ Previous reports have demonstrated that oxidative stress activates JNK and p38 MAPK through apoptosis signal-regulating kinase $1,{ }^{23,} 24$ and JNK is a key modulator in ROS-mediated cell death. ${ }^{25}$ The present study further demonstrated that AOPP-induced ROS led to downstream JNK phosphorylation. The downstream modulatory role of JNK in ROS-mediated cell death is controversial, and involvement of both caspase and PARP-1 pathways have been reported. ${ }^{26-28}$ PARP-1 is an abundant nuclear enzyme that facilitates DNA repair and mediates cell death in ischemia-reperfusion injury, ${ }^{29}$ ROS-induced injury ${ }^{29}$ and inflammatory injury. ${ }^{30,31}$ Our results demonstrated that AOPPs triggered JNK phosphorylation and subsequent PARP-1 activation, followed by PAR formation, large NAD + decreases, and AIF translocation. Although caspase-3 was activated, its activation was not required for AOPP-induced cell death; rather, it may facilitate PARP-1 degradation. Moreover, we also demonstrated that suppression of JNK activation by a chemical inhibitor significantly reduced AOPP-induced PARP-1 activation, suggesting that JNK contributes to sustained PARP-1 activation.

Our findings demonstrated an unexpected pathological effect of AOPPs in inducing inflammatory changes of the intestine, such as shortened villi; inflammatory cell infiltration; and epithelial erosion, necrosis, and exfoliation. Moreover, chronic AOPP-RSA administration to rats reduced goblet cell numbers, suggesting that these cell types are highly susceptible to AOPPs. Paneth cell death may be important in IBD development, ${ }^{15,32}$ but it remains to be investigated whether Paneth cell numbers are reduced after AOPPs treatment. However, pathological alterations induced by AOPPs varied between the ileum and colon tissue. Differences between the two bowel parts implies that intestinal tissue was less resistant to cell death and may provide an explanation for decreased susceptibility of the intestine to neoplastic transformation. In support of this 

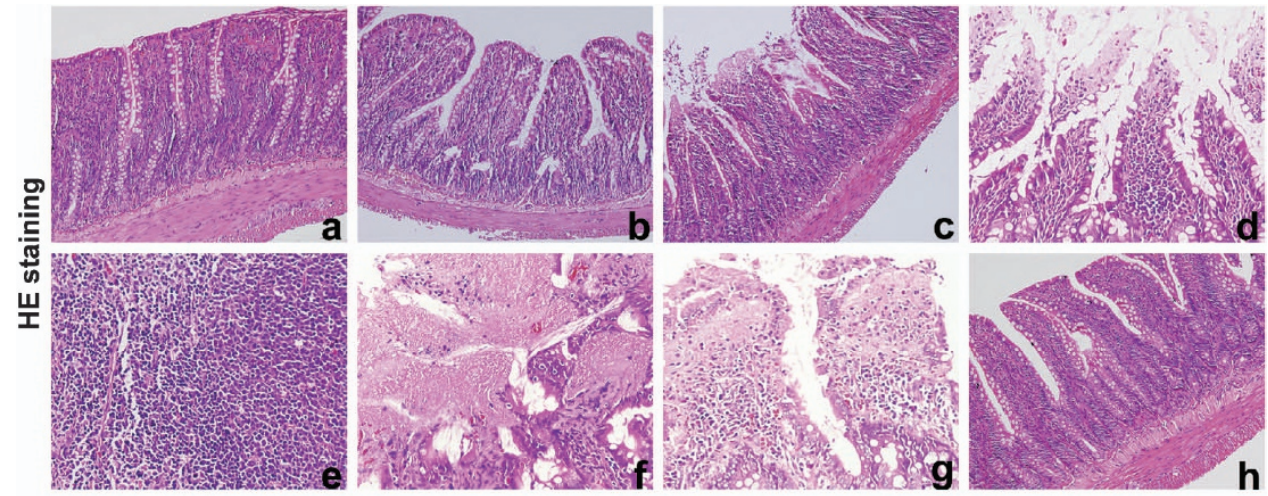

i
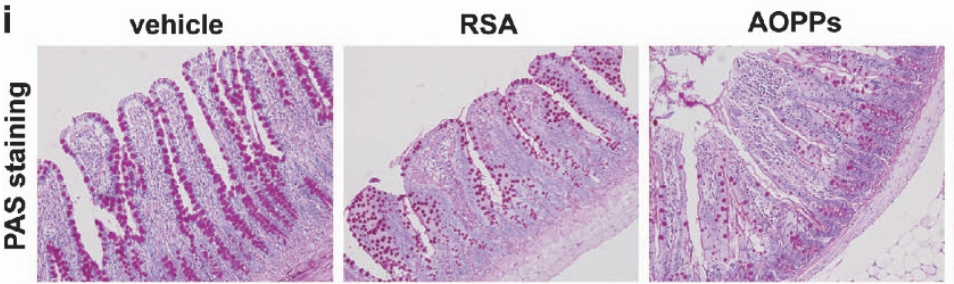

AOPPs+apocynin

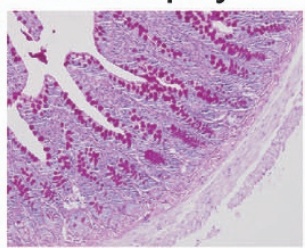

j
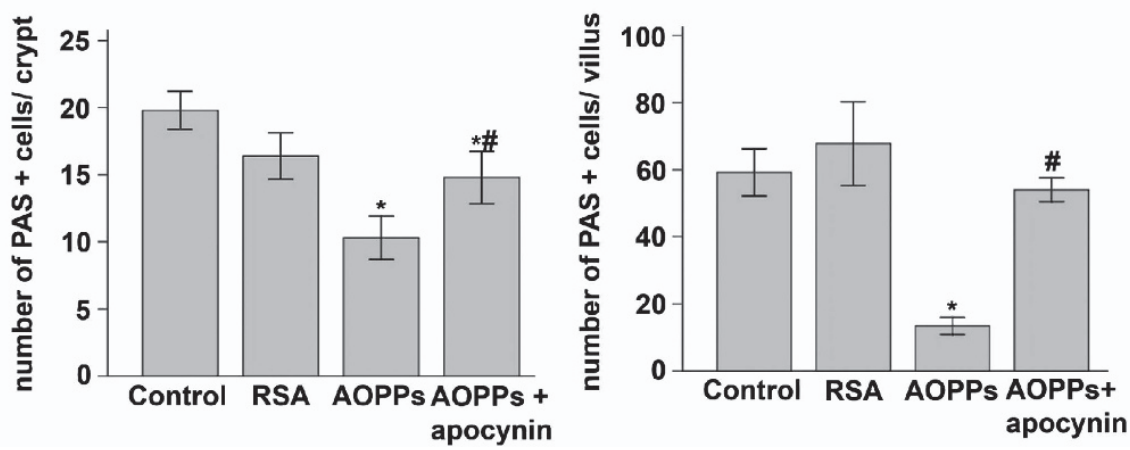

Figure 8 AOPPs treatment of rats induced morphological changes of the small intestinal epithelium and altered the number of goblet cells. H\&E staining showed almost normal intestine in (a) vehicle and (b) RSA groups, whereas (c, d) epithelial erosion and inflammatory cell invasion into the lamina propria and submucosal layer, (e) lymphoid follicle hyperplasia, (f) epithelial necrosis, and (g) epithelial exfoliation were found in AOPP-treated group. (h) Apocynin attenuated the degree of AOPP-induced tissue injury. (i) PAS staining in the small intestines of rats treated with or without AOPPs. (j) Quantification of goblet cells per crypt \pm S.D. of control, RSA, AOPPs, and AOPPs + apocynin group ( $n=6$ per group). ${ }^{*} P<0.05$ versus control. ${ }^{\#}<<0.05$ versus AOPPs

hypothesis, previous studies have shown that stem cells in colonic crypts are more resistant to spontaneous cell death than those in the small intestine. ${ }^{33,34}$

Another group reported that the concentration of circulating AOPPs was elevated in patients with IBD, particularly in those with active CD. ${ }^{17}$ Our study demonstrated enhanced AOPP deposition in IECs, as well as in inflammatory cells in the lamina propria, which were associated with cell death in diseased tissue from patients with CD. The enhancement of AOPPs both in plasma and in the local intestine suggest that AOPPs may affect the intestine through two pathways. Collectively, these results provide important information for further study of the pathogenic effects of AOPPs.

Taken together, our observations are suggestive of a novel mechanism that aggravates IBD: accumulated AOPPs in IBD patients have the capacity to induce ROS generation, which initiates IEC death and intestinal tissue injury. On the basis of the in vivo and in vitro evidence presented in this report, we propose that AOPPs may represent a novel pathogenic factor that contributes to IBD progression. Targeting AOPP-induced cellular mechanisms might emerge as a promising therapeutic option for patients with IBD.

\section{Materials and Methods}

AOPP-RSA preparation and determination. AOPP-RSA was prepared in vitro by incubation of RSA (Sigma, St. Louis, MO, USA) with $\mathrm{HOCl}$ (Fluke, Buchs, Switzerland) as described previously. ${ }^{10}$ Prepared samples were dialyzed against phosphate-buffered saline (PBS) for $24 \mathrm{~h}$ to remove free $\mathrm{HOCl}$ and passed through a Detoxi-Gel column (Pierce, Rockford, IL, USA) to remove contaminated endotoxin. Endotoxin levels in AOPP-RSA were measured with a Limulus Amoebocyte Lysate kit (BioWhittaker, Walkersville, MD, USA) and were found to be below $0.05 \mathrm{ng} / \mathrm{mg}$ protein. AOPPs contents in the preparations were determined with an OxiSelect AOPP Assay Kit (Cell Biolabs, San Diego, CA, USA); AOPPs contents in the AOPP-RSA and unmodified RSA were $50.10 \pm 3.92$ and $0.22 \pm 0.06 \mu \mathrm{mol} / \mathrm{g}$ protein, respectively.

Cell culture. An immortalized rat IEC line (IEC-6, The Committee on Type Culture Collection, Chinese Academy of Sciences, Beijing, China) was cultured in Dulbecco's modified Eagle medium supplemented with $10 \%$ fetal bovine serum, $100 \mathrm{mg} / \mathrm{ml}$ penicillin, and $100 \mathrm{lU} / \mathrm{ml}$ streptomycin in a $5 \%$ carbon dioxide atmosphere at $37^{\circ} \mathrm{C}$. Experiments were performed using passages $10-20$. 
a

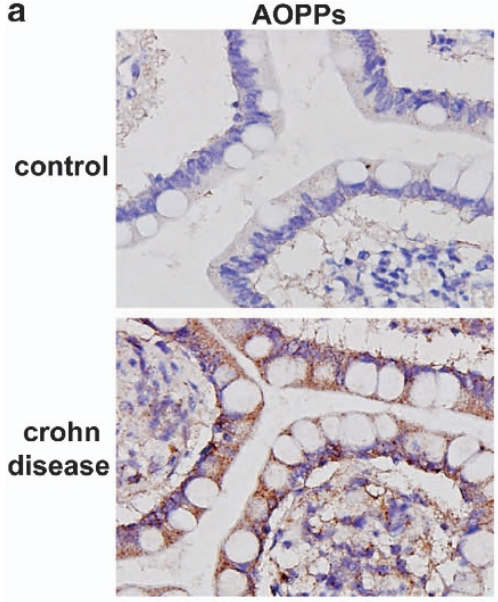

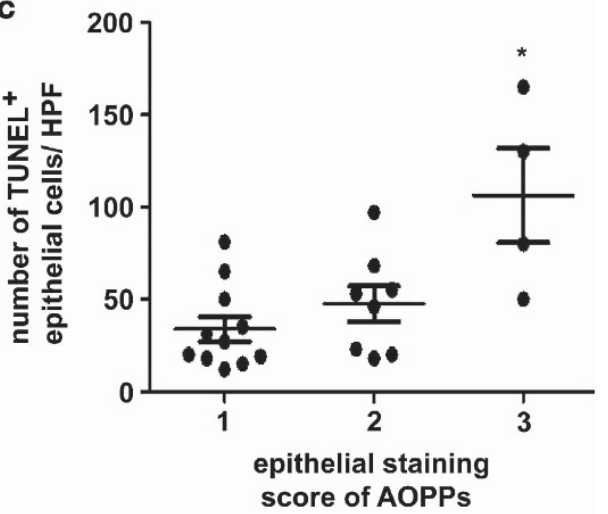

TUNEL
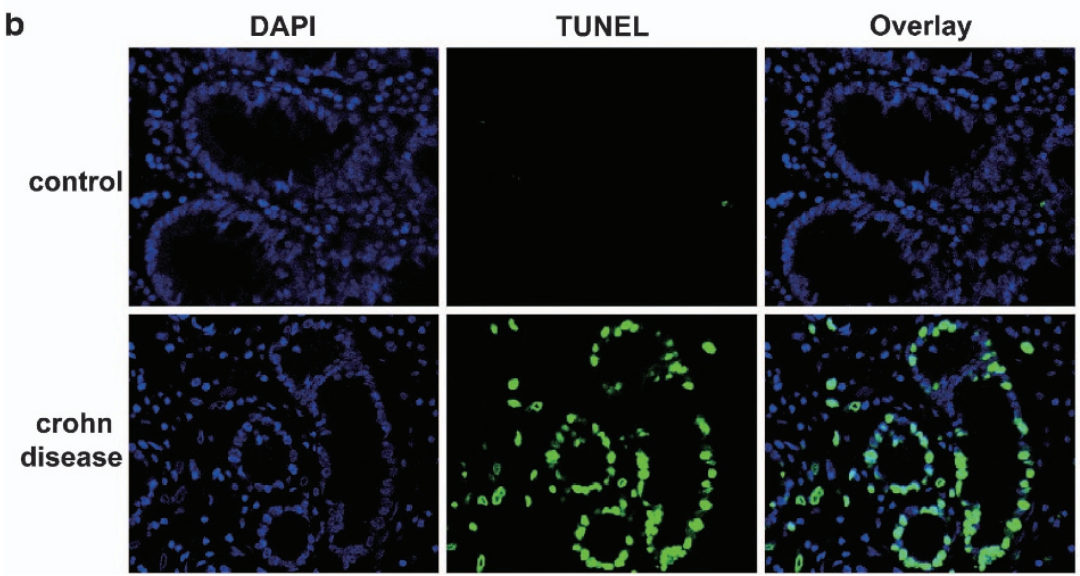

Figure 9 (a) Representative images of AOPPs immunochemistry in paraffin sections of resected intestinal specimens from patients with $C D(n=23)$. Normal tissue adjacent to the diseased intestine was used as a normal control. (b) Immunofluorescence TUNEL labeling in small intestinal epithelium sampled from patients with CD. (c) The high AOPPs immunoreactivity score revealed an increased number of apoptotic cells. HPF: high-power fields. ${ }^{*}<0.05$ versus control

Apoptosis assays in IEC-6 cultures. Assessment of FITC annexin V-labeled apoptotic cells was performed according to the protocol provided by the manufacturer (Becton Dickinson, Franklin Lakes, NJ, USA). Cells were seeded on six-well plates and treated with or without AOPP-RSA for the indicated time; cells $\left(1 \times 10^{6}\right)$ were suspended in buffer containing FITC annexin V and PI. The samples were analyzed with a FACS Calibur flow cytometer (Becton Dickinson). A total of 10000 cells were analyzed per determination. Cells were considered apoptotic if they were undergoing either early (Annexin-V-positive, PI-negative) or late apoptosis (Annexin-V-positive, PI-positive).

Determination of ROS generation. Intracellular ROS generation was measured with a flow cytometer (Becton Dickinson) with the probe DCFH-DA $\left(2^{\prime}, 7^{\prime}\right.$-DCF-diacetate), which is a cell-permeable, non-fluorescent dye that can be oxidized to the fluorescent $2^{\prime}, 7^{\prime}$-DCF by ROS inside cells. Briefly, IEC-6 cultures were incubated with $10 \mu \mathrm{M}$ DCFH-DA for $30 \mathrm{~min}$ at $37^{\circ} \mathrm{C}$ followed by AOPPs treatment as described above.

Western blotting. Cultured cells or frozen rat intestinal tissue samples were lysed in radio-immunoprecipitation assay buffer, and protein was collected after centrifugation and mixed with $5 \times$ sodium dodecyl sulfate (SDS) sample buffer. The samples were separated by SDS-polyacrylamide gel electrophoresis (PAGE) using $8-12 \%$ acrylamide gels and then transferred to polyvinylidene fluoride (PVDF) membranes (Millipore, Billerica, MA, USA). After incubation with primary and secondary antibodies, the protein bands were detected with chemiluminescence detection reagents (Millipore). The following antibodies (Abs) were used: goat anti-p22 ${ }^{\text {phox }}$, goat anti-gp91 ${ }^{\text {phox }}$ pAb, and goat anti-p47 ${ }^{\text {phox }}$ pAbs were all from Santa Cruz Biotechnology (Santa Cruz, CA, USA); anti-PARP-1 pAb, anti$\mathrm{Bcl}-2 \mathrm{pAb}$, anti-Bax pAb, anti-caspase-3 pAb, anti-JNK Ab, and anti-pJNK Ab were from Cell Signaling Technology (Beverly, MA, USA); anti-PAR mAb was from Millipore; rabbit anti-P47 ${ }^{\text {phox }}$ pAb was from Sigma; and anti-AIF Ab was from Abcam (Cambridge, UK). Mouse anti-AOPP Ab was a gift from professor Fu Ning (Southern Medical University, Guangzhou, China). Mouse anti- $\beta$-actin Ab and goat anti-mouse, rabbit anti-goat, and goat anti-rabbit IgG-horseradish peroxidase (HRP) were purchased from Boster (Wuhan, China).

p47 $7^{\text {phox }}$ phosphorylation. $\mathrm{p} 47^{\text {phox }}$ phosphorylation in IEC- 6 cultures was measured by immunoprecipitation as described previously. ${ }^{18}$ Briefly, cell lysates were incubated with protein $A / G$ agarose beads (Santa Cruz Biotechnology), and a polyclonal rabbit anti-phosphoserine Ab (Abcam). The precipitated immunocomplexes were resolved by SDS-PAGE, transferred onto PVDF membranes (Millipore), incubated with an HRP-conjugated rabbit anti-p4 $7^{\text {phox }}$ antibody (Sigma), and subjected to chemiluminescence detection as described above.

Immunofluorescence staining. $\mathrm{p} 47^{\text {phox }}$ translocation from the cytoplasm to the membrane and AIF migration were detected using immunofluorescence staining. Cells were fixed with paraformaldehyde, washed, and permeabilized with $0.1 \%$ Triton X-100 for $20 \mathrm{~min}$. After blocking with non-fat milk for $1 \mathrm{~h}$, the cells were incubated with anti-p47 $7^{\text {phox }}$ or anti-AIF Ab overnight at $4{ }^{\circ} \mathrm{C}$. The cells were then incubated with Alex 555-conjugated donkey anti-goat IgG (Invitrogen, Carlsbad, $\mathrm{CA}, \mathrm{USA}$ ) or rhodamine-conjugated chicken anti-rabbit IgG-R, stained with DAPI (4',6-diamidino-2-phenylindole), and observed under an OLYMPUS XB-51 fluorescence inverted microscope (Olympus, Tokyo, Japan).

Nuclear/cytosolic fractionation. Subfractionation was performed using a Nuclear/Cytosolic Fractionation Kit (Beyotime, Wuhan, China). IEC-6 cultures were washed with ice-cold PBS, scraped from the plates, and collected. After 
centrifugation, the supernatant was discarded, and the cells were suspended with Cytosol Extraction Buffer containing DTT/protease inhibitors, incubated on ice for $10 \mathrm{~min}$, and Cell Lysis Reagent was added. The nuclei fraction was fractioned at $800 \times \mathrm{g}$ for $10 \mathrm{~min}$. The supernatant was further centrifuged at $12000 \times \mathrm{g}$ for $10 \mathrm{~min}$, and the final supernatant was collected for cytoplasmic fraction. The nuclei pellet was washed and resuspended with Nuclear Extraction Buffer containing DTT/protease inhibitors.

Animal studies. The protocols of this study were approved by the Laboratory Animal Care and Use Committee of Southern Medical University. Male Sprague Dawley rats (initial weight, $160-200 \mathrm{~g}$, Southern Medical University Animal Experiment Center, Guangzhou, China) were housed in a pathogen-free environment and allowed free access to water and diet. The rats were randomly divided into four groups containing six animals per group and received daily intraperitoneal injections of vehicle (PBS, pH 7.4), unmodified RSA (50 mg/kg per day), AOPP-RSA ( $50 \mathrm{mg} / \mathrm{kg}$ per day), or AOPP-RSA (50 mg/kg per day) with or without separate intragastric administration of NADPH oxidase inhibitor apocynin (Sigma, $50 \mathrm{mg} / \mathrm{kg}$ per day). AOPP-RSA dosages were based on our preliminary experiment indicating that by this procedure, plasma AOPP concentrations in the AOPP-RSA-treated group increased $\sim 0.5$-fold compared with the vehicle group (the level that has been found in IBD patients). ${ }^{17}$ At the end of 4,8 , or 12 weeks, rats were anesthetized with sevoflurane and exsanguinated. The duodenum, jejunum, and ileum were collected, flushed with ice-cold PBS, and stored for further analyses.

H\&E staining, PAS staining, and immunohistochemistry. Duodenum, jejunum, and ileum tissues were separately removed and fixed in neutral-buffered formalin. Formalin-fixed specimens were embedded in paraffin, cut into 3-4- $\mu \mathrm{m}$-thick transverse sections, and stained with hematoxylin and eosin (H\&E) to assess epithelial morphology and eosinophilic infiltration. PAS staining was performed according to standard protocol using PAS Staining System reagents from Sigma.

For immunohistochemistry studies, after antigen retrieval, endogenous peroxidase activity, and normal serum blocking, the sections were incubated with primary antibody overnight followed by biotinylated secondary antibodies (Zhongshanjinqiao, Beijing, China). Proteins were visualized as brown pigments via a standard diaminobenzidine (Zhongshanjinqiao) protocol. The slides were lightly counterstained with hematoxylin.

Apoptosis assays of intestinal tissues. Apoptotic cells in the intestinal tissue sections were assessed with TUNEL assays (In situ cell death detection kit, Roche, Mannheim, Germany). Briefly, tissue sections were incubated with proteinase $\mathrm{K}$ for $20 \mathrm{~min}$ at room temperature and then washed with PBS. After inactivating endogenous peroxidase, sections were incubated in TdT buffer containing FITC-conjugated dUTP at $37^{\circ} \mathrm{C}$ for $60 \mathrm{~min}$. Morphological nuclear changes were observed by counterstaining with DAPI (Beyotime). The sections were analyzed under a confocal microscope (Carl Zeiss, Inc., Oberkochen, Germany). The apoptotic cells were counted in five random high-power fields (HPF, each 300 cells), and a total of 1500 epithelial cells were counted. The positive cells were scored for apoptosis. Data were expressed as numbers of apoptotic cells/HPF.

Table 2 Characteristics of patients with CD $(n=23)$

\begin{tabular}{lc}
\hline Parameter & \\
\hline Gender & \\
$\quad$ Male & 20 \\
Female & 3 \\
Age (years) & $35.61 \pm 14.30$ \\
& \\
Disease location & 12 \\
Small bowel & 11 \\
Small bowel and colon & $34.74 \pm 32.45$ \\
CRP & $34.08 \pm 28.37$ \\
ESR & \\
\hline
\end{tabular}

Abbreviations: CRP, C-reactive protein; ESR, erythrocyte sedimentation rate Data are expressed as mean \pm S.D.
Patients and specimens. A total of 23 formalin-fixed, paraffin-embedded intestinal resection specimens from CD patients who underwent segmental small bowel resection were obtained from the Nanfang hospital of Southern Medical University (Guangzhou, China) from 2010 to 2012. The diagnosis of CD was based on established clinical and histologic criteria. Patients with malignant tumor, cardiovascular disease, severe infection, or infliximab use were excluded. Normal intestinal tissue adjacent to diseased tissue was used as normal control. This study was approved by the Medical Ethical Committee of Nanfang hospital, and specimens were treated anonymously according to ethical and legal standards. Patient demographic data are presented in Table 2.

Statistical analysis. All experiments were repeated at least three times. Continuous variables are expressed as mean \pm standard deviation (S.D.). For multiple comparisons within a data set, one-way analysis of variance with least significant difference or Dunnett's T3 test was performed. A two-tailed $P$-value of $<0.05$ was considered statistically significant. Statistical analyses were performed with SPSS 13.0 software (SPSS Inc., Chicago, IL, USA).

\section{Conflict of Interest}

The authors declare no conflict of interest.

Acknowledgements. This work was supported by grants from the National Natural Science Foundation (81170354), the Guangdong Provincial Science and Technology Plan Fund (2011B031800195), and the Natural Science Foundation of Guangdong Province (S2012010009343).

1. Loftus EV Jr. Clinical epidemiology of inflammatory bowel disease: incidence, prevalence, and environmental influences. Gastroenterology 2004; 126: 1504-1517.

2. Zhu H, Li YR. Oxidative stress and redox signaling mechanisms of inflammatory bowel disease: updated experimental and clinical evidence. Exp Biol Med 2012; 237: 474-480

3. Kruidenier L, Kuiper I, Van Duijn W, Mieremet-Ooms MA, van Hogezand RA, Lamers CB et al. Imbalanced secondary mucosal antioxidant response in inflammatory bowel disease. J Pathol 2003; 201: 17-27.

4. Dean RT, Fu S, Stocker R, Davies MJ. Biochemistry and pathology of radical-mediated protein oxidation. Biochem J 1997; 324: 1-18

5. Witko-Sarsat V, Friedlander M, Nguyen Khoa T, Capeillere-Blandin C, Nguyen AT, Canteloup $\mathrm{S}$ et al. Advanced oxidation protein products as novel mediators of inflammation and monocyte activation in chronic renal failure. J Immunol 1998; 161: 2524-2532.

6. Witko-Sarsat V, Friedlander M, Capeillere-Blandin C, Nguyen-Khoa T, Nguyen AT, Zingraff $\mathrm{J}$ et al. Advanced oxidation protein products as a novel marker of oxidative stress in uremia. Kidney Int 1996; 49: 1304-1313.

7. Kalousova M, Skrha J, Zima T. Advanced glycation end-products and advanced oxidation protein products in patients with diabetes mellitus. Physiol Res 2002; 51: 597-604.

8. Ozenirler S, Erkan G, Gulbahar O, Bostankolu O, Ozbas Demirel O, Bilgihan A et al. Serum levels of advanced oxidation protein products, malonyldialdehyde, and total radical trapping antioxidant parameter in patients with chronic hepatitis C. Turk J Gastroenterol 2011; 22: 47-53

9. Guo ZJ, Niu HX, Hou FF, Zhang L, Fu N, Nagai R et al. Advanced oxidation protein products activate vascular endothelial cells via a RAGE-mediated signaling pathway. Antioxid Redox Signal 2008; 10: 1699-1712.

10. Zhou LL, Cao W, Xie C, Tian J, Zhou Z, Zhou Q et al. The receptor of advanced glycation end products plays a central role in advanced oxidation protein products-induced podocyte apoptosis. Kidney Int 2012; 82: 759-770.

11. Zhong ZM, Bai L, Chen JT. Advanced oxidation protein products inhibit proliferation and differentiation of rat osteoblast-like cells via NF-kappaB pathway. Cell Physiol Biochem 2009; 24: 105-114.

12. Marchiando AM, Shen L, Graham WV, Edelblum KL, Duckworth CA, Guan Y et al. The epithelial barrier is maintained by in vivo tight junction expansion during pathologic intestinal epithelial shedding. Gastroenterology 2011; 140: 1208-1218.

13. Artis D. Epithelial-cell recognition of commensal bacteria and maintenance of immune homeostasis in the gut. Nat Rev Immunol 2008; 8: 411-420.

14. Gunther C, Neumann H, Neurath MF, Becker C. Apoptosis, necrosis and necroptosis: cell death regulation in the intestinal epithelium. Gut 2012; 62: 1062-1071.

15. Gunther C, Martini E, Wittkopf N, Amann K, Weigmann B, Neumann H et al. Caspase-8 regulates TNF-alpha-induced epithelial necroptosis and terminal ileitis. Nature 2011; 477: 335-339.

16. Chiarugi A, Moskowitz MA. PARP-1-a perpetrator of apoptotic cell death? Science 2002; 297: 200-201.

17. Krzystek-Korpacka M, Neubauer K, Berdowska I, Boehm D, Zielinski B, Petryszyn P et al. Enhanced formation of advanced oxidation protein products in IBD. Inflamm Bowel Dis 2008; 14: 794-802 
18. Baskol M, Baskol G, Kocer D, Ozbakir O, Yucesoy M. Advanced oxidation protein products: a novel marker of oxidative stress in ulcerative colitis. J Clin Gastroenterol 2008; 42 : 687-691.

19. Son Y, Kim S, Chung HT, Pae HO. Reactive oxygen species in the activation of MAP kinases. Meth Enzymol 2013; 528: 27-48.

20. Almenier HA, Al Menshawy HH, Maher MM, Al Gamal S. Oxidative stress and inflammatory bowel disease. Front Biosci (Elite Ed) 2012; 4: 1335-1344.

21. Zhou LL, Hou FF, Wang GB, Yang F, Xie D, Wang YP et al. Accumulation of advanced oxidation protein products induces podocyte apoptosis and deletion through NADPH-dependent mechanisms. Kidney Int 2009; 76: 1148-1160.

22. Circu ML, Aw TY. Intestinal redox biology and oxidative stress. Semin Cell Dev Biol 2012; 23: 729-737.

23. Goldman EH, Chen L, Fu H. Activation of apoptosis signal-regulating kinase 1 by reactive oxygen species through dephosphorylation at serine 967 and 14-3-3 dissociation. J Biol Chem 2004; 279: 10442-10449.

24. Bhattacharyya S, Dudeja PK, Tobacman JK. ROS, Hsp27, and IKKbeta mediate dextran sodium sulfate (DSS) activation of IkappaBa, NFkappaB, and IL-8. Inflamm Bowel Dis 2009; 15: 673-683.

25. Shen HM, Liu ZG. JNK signaling pathway is a key modulator in cell death mediated by reactive oxygen and nitrogen species. Free Radic Biol Med 2006; 40: 928-939.

26. Yu SW, Wang H, Poitras MF, Coombs C, Bowers WJ, Federoff HJ et al. Mediation of poly(ADP-ribose) polymerase-1-dependent cell death by apoptosis-inducing factor. Science 2002; 297: 259-263.

27. Susztak K, Raff AC, Schiffer M, Bottinger EP. Glucose-induced reactive oxygen species cause apoptosis of podocytes and podocyte depletion at the onset of diabetic nephropathy. Diabetes 2006; 55: 225-233.
28. Zhang S, Lin Y, Kim YS, Hande MP, Liu ZG, Shen HM. c-Jun N-terminal kinase mediates hydrogen peroxide-induced cell death via sustained poly(ADP-ribose) polymerase-1 activation. Cell Death Differ 2007; 14: 1001-1010.

29. van Wijk SJ, Hageman GJ. Poly (ADP-ribose) polymerase-1 mediated caspaseindependent cell death after ischemia/reperfusion. Free Radic Biol Med 2005; 39 81-90.

30. Zingarelli B, Hake PW, Burroughs TJ, Piraino G, O'Connor M, Denenberg A. Activator protein-1 signalling pathway and apoptosis are modulated by poly(ADP-ribose) polymerase-1 in experimental colitis. Immunology 2004; 113: 509-517.

31. Ba X, Garg NJ. Signaling mechanism of poly(ADP-ribose) polymerase-1 (PARP-1) in inflammatory diseases. Am J Path 2011; 178: 946-955.

32. McSorley SJ, Bevins CL. Paneth cells: targets of friendly fire. Nat Immunol. 2013; 14: 114-116.

33. Watson AJ, Pritchard DM. Lessons from genetically engineered animal models. VII. Apoptosis in intestinal epithelium: lessons from transgenic and knockout mice. Am J Physiol Gastrointest Liver Physiol 2000; 278: G1-G5.

34. Renehan AG, Bach SP, Potten CS. The relevance of apoptosis for cellular homeostasis and tumorigenesis in the intestine. Can J Gastroenterol 2001; 15: 166-176.

(c) (1) (2) Cell Death and Disease is an open-access journal published by Nature Publishing Group. This work is licensed under a Creative Commons Attribution-NonCommercialShareAlike 3.0 Unported License. To view a copy of this license, visit http://creativecommons.org/licenses/by-nc-sa/3.0/ 